\title{
Perceptions of New Graduate Chiropractors in Their Management of Paediatric Patients in the eThekwini Municipality
}

\author{
Tarryn Miranda Frederick ${ }^{1}$, Desiree Varatharajullu ${ }^{1} \&$ Maureen Nokuthula Sibiya ${ }^{2}$ \\ ${ }^{1}$ Department of Chiropractic, Durban University of Technology, Ritson Campus, Durban, South Africa \\ ${ }^{2}$ Faculty of Health Sciences, Durban University of Technology, Ritson Campus, Durban, South Africa \\ Correspondence: Tarryn Miranda Frederick, Department of Chiropractic Durban University of Technology, P.O. \\ Box 1334, Durban, 4000, South Africa. Tel: 27-62- 978-4593. E-mail: tmfrederick94@gmail.com
}

\author{
Received: October 9, 2019 Accepted: November 18, 2020 Online Published: February 15, 2020 \\ doi:10.5539/gjhs.v12n3p32 URL: https://doi.org/10.5539/gjhs.v12n3p32
}

\begin{abstract}
The aim of the study was to assess the perceptions of new graduate chiropractors in their management of paediatric patients in the eThekwini Municipality. A qualitative, explorative, descriptive research design was utilised for this study. A purposive sample of 10 new graduate chiropractors in the eThekwini Municipality were interviewed. Data was collected utilising semi-structured interviews. The collected data was then stored electronically and transcribed. The transcripts were then coded and analysed by the researcher to identify categories and subcategories. The coded data was thereafter interpreted, using the themes and sub-themes identified. Four main themes were identified in the study, namely self-perception of confidence, knowledge and skill in paediatric patient management; exposure and training received in the chiropractic course; challenges encountered in paediatric patient management; and further enhancement of paediatric patient management. New graduate chiropractors expressed a lack of confidence and competence with regards to their management of the paediatric population, which was found to correlate with their lack of exposure to paediatric patients during the chiropractic paediatric curriculum in the course. Participants stated that their lack of exposure to this patient group negatively impacted on their diagnostic ability, orthopaedic assessment and treatment of paediatric patients. Participants identified areas of improvement in the chiropractic paediatric curriculum. Methods to enhance paediatric assessment and management were then discussed further. The findings of this study indicate that lack of exposure to paediatric patients prior to private practice was a key factor that negatively impacted on a new graduate's confidence to assess, treat and manage paediatrics.
\end{abstract}

Keywords: Chiropractic, education, new graduate chiropractors, paediatric patients, perceptions.

\section{Introduction}

The chiropractic management of paediatric patients remains a controversial area in the health care sector; however, chiropractors still remain the most popular complementary alternative medicine providers for children and adolescents (Ferrance \& Miller, 2010). A paediatric patient, in the context of this study, refers to anyone up to and including the age of 18 years old (Children's Institute, 2016). Chiropractors provide safe and effective therapeutic interventions to the paediatric population as an alternative option to drug and surgical interventions (Vallone, 2018). The Chiropractic profession focuses on the relationship between the structure and function of the body and the manner in which this relationship affects the preservation and restoration of health (Vallone, 2018). In South Africa, chiropractors employ a holistic approach to patient care, not only focusing on the diagnosis, treatment and prevention of neuromuscularskeletal disorders, but also serving as a valuable resource in injury prevention, nutritional counselling, postural correction, stress management and work-place safety (Hart, 2016).

Paediatric visits to chiropractors encompass a diverse range of musculoskeletal and non-musculoskeletal disorders which include, but are not limited to: torticollis, traumatic injuries, back pain, Bell's palsy, respiratory disorders and attention deficit disorder amongst others (Ferrance \& Miller, 2010). As a result of the anatomical, physiological and psychological variance between adults and children, the management of paediatric patients must be modified and age-appropriate (Davies, 2000). Due to an immature musculoskeletal system, constant bodily dynamic changes, congenital defects and developmental disorders, the presentation and management of certain illnesses may create doubt and confusion among many new graduate chiropractors (Mortazavi et al., 2001). Ethical considerations regarding informed consent, privacy and guardianship must also be taken into consideration at 
every consult (Maharaj, 2017). The chiropractic management of children therefore, requires a multi-faceted approach in combination with competence and confidence on behalf of the chiropractor.

Chiropractic clinical education and training prepares the chiropractic student to assess, manage or co-manage paediatric patients, however, paucity in the literature exists regarding the perceived standard of education received and the ability of the basic paediatric course taught at Master's degree level, to substantially prepare future chiropractors in paediatric patient management. By assessing the perceptions of new graduate chiropractors in their management of paediatric patients in the eThekwini Municipality with regards to their knowledge, confidence and skill in paediatric management, areas of enhancement in chiropractic paediatric educational development and training can be identified and addressed. A new graduate chiropractor, in the context of this study, is defined as a graduate obtaining their Master's degree in Chiropractic and obtaining employment for five years or less (Bhatia, 2015). The research was conducted with new graduate chiropractors, with the aim of eliminating experience, which is a key contributor to the development of knowledge and perception (Todd, Carrol, Russel \& Mitchell, 2017).

In South Africa, the Chiropractic course is offered only at the two institutions, one being a University of Technology in Durban in the province of KwaZulu-Natal (KZN) and the other being a University in Johannesburg, Gauteng. At the University of Technology in Durban, the chiropractic academic programme spans over a five year period, with an additional year consisting of the chiropractic students' internship. Undergraduate training comprises of three years of full time study, providing the chiropractic student with an extensive foundation based on traditional medical subjects, emphasizing the development of diagnostic skills. A combination of lectures, tutorials, clinical and laboratory work constitute the basic instructional pattern. The latter two years of the chiropractic programme focuses on neuromusculoskeletal disorders and holistic patient care. The fifth year encompasses a theoretical component with the introduction of clinical experience (DUT, 2018).

The paediatric component of the course is integrated into the Clinical Chiropractic module and completed within the fifth academic year of the Chiropractic programme. The paediatric module overviews the following:

- History taking, assessment, diagnosis and treatment of basic paediatric conditions.

- Understanding of the modalities used to evaluate and treat paediatrics.

- Paediatric nutrition

- Paediatric rehabilitation

- Exercise prescription and paediatric emergency protocols.

By investigating the perceptions of new graduate chiropractors in their management of paediatric patients in the eThekwini Municipality, in the province of KZN, the conclusions drawn from the study can provide a premise for the understanding of methods in which knowledge; skill and confidence can be further enhanced during tertiary education.

\section{Methods}

\subsection{Research Design}

A qualitative, exploratory, descriptive design was used for this study. A qualitative paradigm was useful in order to gain an understanding of human behaviour, with the aim of describing and exploring the nature and quality of how people understand, experience and behave, which aligns with the aim of this study. The researcher utilised semi-structured interviews to gain insight into new graduates' perceptions of their management of paediatric patients, with the research design allowing the participants to provide unique, institutional perspectives and background information. The researcher was able to explore and interpret concepts, while identifying relevance and meaning from the collected data.

\subsection{Research Setting}

The participants for this study were sourced from the eThekwini Municipality which is located in the province of KZN. The municipal area spans across $2297 \mathrm{~km}^{2}$ and is home to approximately 3.5 million people (South Africa, Department of Infrastructure, 2012).

\subsection{Population and Sampling Process}

The study population consisted of 10 new graduate chiropractors who had graduated from the University of Technology in Durban and obtained five years or less of employment. The researcher utilised purposive sampling in order to select the sample. This is a technique whereby the researcher's judgement is utilised to choose the participants to include in the study (Dudovsky, 2016). New graduate chiropractors were identified with the aid of 
the supervisor using a list obtained from the Allied Health Professions Council of South Africa (AHPCSA). Participants were then contacted telephonically in order to obtain permission to conduct the study at the participant's place of employment.

\subsection{Data Collection}

Data was collected by conducting semi-structured interviews with the use of an interview guide. The duration of the interviews averaged approximately 15 minutes. The researcher began the interviews by explaining the context and purpose of the study and obtaining permission for the interview to be voice recorded. A letter in information and consent was then provided to the participants, which was read, signed and returned to the researcher. The researcher then emphasized that participation was voluntary and assured participants of confidentiality. A set of eight research questions, with additional probing questions were then asked and participants were allocated time to answer each question.

\subsection{Data Analysis}

The data was analysed as outlined by Creswell, (2013), into three phases, namely transcription, analysis and interpretation. Transcription occurred after listening to the voice recordings from interviews verbatim. The transcripts and voice recordings were thereafter rechecked to ensure accuracy. Transcripts were coded and analysed to identify categories and subcategories. By utilising the themes and subthemes identified by the researcher, the coded data was then interpreted. The interpretations were thereafter rechecked by the research supervisor to reduce bias. Conclusions were then formulated when similar themes were clustered together.

\subsection{Trustworthiness}

Reliable research methods utilised in other qualitative studies were used during the research process. Credibility was ensured by issuing a letter of consent to all participants. Data was collected until the point of saturation occurred. Transferability was ensured by providing the participants with a letter of information and allowing aspects of the research process, such as the methods of data collection, to be provided to the participants. Dependability was accomplished by tracking the research design and keeping an audit trail. Conformability was accomplished in the study by maintaining subjectivity (Morrow, 2005).

\subsection{Ethical Considerations}

Ethical approval was obtained from the Institutional Research and Ethics Committee (IREC 133/18). Each participant read and signed the letter of information and consent prior to commencement of the interview at their place of employment. To ensure confidentiality, the participant's personal details were omitted form the transcripts and recordings, which were only accessible by the researcher, supervisor and co-supervisor.

\section{Results}

The themes and sub-themes that emerged from the analysis of the interviews are presented along with excerpts from the transcripts in order to substantiate the results. Table 1 presents an overview of the emergent themes and sub-themes reflecting the perceptions of new graduate chiropractors in their management of paediatric patients in the eThekwini Municipality. 
Table 1. Themes and sub-themes reflecting the perceptions of new graduate chiropractors in their management of paediatric patients in the eThekwini Municipality

\begin{tabular}{|c|c|}
\hline Theme 1 & Self-perception of confidence, knowledge and skill in paediatric patient management \\
\hline Sub-theme 1.1 & Confidence and knowledge in the management of different paediatric age categories. \\
\hline Sub-theme 1.2 & Inter-professional relationships and referrals to other chiropractors/healthcare specialists. \\
\hline Theme 2 & Paediatric exposure and training received in the chiropractic course \\
\hline Sub-theme 2.1 & Experience in the diagnostic and orthopaedic assessment of a paediatric. \\
\hline Sub-theme 2.2 & Experience in paediatric patient adjustment and auxiliary treatment protocol. \\
\hline Sub-theme 2.3 & Beneficial aspects learnt during the chiropractic paediatric module. \\
\hline Sub-theme 2.4 & $\begin{array}{l}\text { Additional sources of information utilised to facilitate greater knowledge consolidation during the } \\
\text { chiropractic paediatric module. }\end{array}$ \\
\hline Sub-theme 2.5 & Recommended areas of improvement in the chiropractic paediatric module. \\
\hline Theme 3 & Challenges encountered in paediatric patient management \\
\hline Sub-theme 3.1 & Parental satisfaction and influence on treatment protocol. \\
\hline Sub-theme 3.2 & Communication barrier with the younger paediatric population. \\
\hline Sub-theme 3.3 & Uncooperative paediatric patients. \\
\hline Sub-theme 3.4 & Time management. \\
\hline Sub-theme 3.5 & Knowledge, confidence and practical experience in paediatric patient management. \\
\hline Theme 4 & Enhancement of paediatric management \\
\hline Sub-theme 4.1 & Improvement in paediatric assessment. \\
\hline Sub-theme 4.2 & Improvement in paediatric treatment. \\
\hline Sub-theme 4.3 & Continuation of professional development in paediatrics. \\
\hline
\end{tabular}

\subsection{Theme one: Self-perception of Confidence, Knowledge and Skill in Paediatric Patient Management}

A lack of confidence and knowledge was expressed by new graduate chiropractors when managing the paediatric population. A strong correlation was exhibited between self-perceived skill in paediatric management and the referral rate of paediatric patients.

\section{Confidence and knowledge in the management of different paediatric age categories}

New graduate chiropractors voiced a lack of confidence and knowledge when managing the younger paediatric population, especially children that could not verbally communicate. This is substantiated by a participant who stated that: "I don't think so hey (with regards to being knowledgeable to diagnose a paediatric patient). I think with infants, probably not, uhm... With like slightly older kids that can talk, then yes, because obviously you can get a feel of like asking them what sort of pain it is and where they feel the pain. Obviously with infants we wouldn't know that because they just cry all the time..." (Participant 2)

Another participant voiced a similar opinion, stating the following: "I would say up until the age of probably ten, (participant pauses) ten and older, I think I'm quite adequate in dealing with, but I think when you start getting to the real paediatrics, sort of your few month-old babies to your few years old, I don't think from varsity that we are prepared for that. I would say the younger patients, under ten, it's very, very difficult to diagnose accurately and say a $100 \%$ without getting a referral to a specialist to confirm." (Participant 4)

\section{Inter-professional relationships and referrals to other chiropractors/ healthcare specialists}

Participants expressed a need to seek advice from chiropractors with more experience and refer to other healthcare specialists or chiropractors in their management protocol of paediatrics. This is confirmed by a participant who said that: "So, in my very first experience of a young paediatric patient was seven days old, where I couldn't very well use Diversified technique as they are used in adults. So what I did was, I picked up the phone, spoke to two practitioners that have been in practice for over ten years and when I saw there was a booking for later that day, I went in and got some insight..." (Participant 1) 
Another participant stated that: "I think we can't work in isolation, especially with babies. As I said earlier, I often wait for the diagnosis from the paediatrician first, to say that the baby has infantile colic and that the parents are er, you know, seeking alternatives." (Participant 5)

\subsection{Theme two: Paediatric exposure and training received in the Chiropractic course}

Diverse opinions were expressed regarding the lecturing and training received during the Chiropractic paediatric module. General discontent was exhibited towards the level of diagnostic and therapeutic technique training as well as lecturing style and the theoretical component content.

\section{Experience in the diagnostic and orthopaedic assessment of a paediatric}

Participants believed that there was a substantial paucity in the practical component of diagnostic and orthopaedic assessment taught in the chiropractic paediatric module. The following excerpts from participants substantiated this:

"More time, more practicals, uhm, a little bit more focus on paediatrics. Ifound personally, that it was one of those subjects that was very basic and brushed over at varsity. It was pretty much. "Here's the book, go and learn it and then we'll test you on that." There were no practical aspects. I think the only practical stuff we did were the reflexes in varsity and even those were your basic-basic sort of test. So, I definitely think we need to go into more detail, sort of know more conditions regarding actual paediatrics. So, we didn't even do paediatric $x$-rays or any diagnostic imaging...” (Participant 4)

"We didn't know how to, I'll say look at, consider, diagnose and treat a paediatric patient, other than what we had already been taught with the standard patient and adult." (Participant 1)

\section{Experience in paediatric patient adjustment and auxiliary treatment protocol}

Participants were of the opinion that there was a void in their capability to perform adjustments and auxiliary treatment on paediatric patients due to lack of frequent practical sessions focusing on these aspects, in the paediatric module. This is supported by the participants who stated the following:

"When I first treated a three-year-old, I felt very nervous, because they feel fragile and we don't have much practice in clinic and neither did we have any practicals treating paediatrics." (Participant 8)

Another participant who shared a similar opinion said: "So, uhm, the course that we did, obviously was not sufficient in order to treat a paediatric patient sufficiently. Uhm, and then obviously modifying your treatment, form how you would treat an older patient, to how you would treat a baby. I would say that there's a lot that would need to be modified and if you are not confident in that case, you're going to be less inclined to want to treat those patients." (Participant 9)

\section{Beneficial aspects learnt during the Chiropractic paediatric module}

Diverse views were expressed with regards to the most beneficial aspect learnt in the paediatric module, which aided in the treatment and management of paediatrics in private practice. The participants' responses reflected these disparities: A participant that felt that their theoretical grounding was the most useful aspect in the paediatric module said that:

“...I think that we have really good theory grounding, it's just that we lack the practical aspect.” (Participant 10)

Another participant shared a similar opinion; however felt that there was still paucity in the theoretical grounding offered by the Chiropractic course, which is supported by the following excerpt:

"The most useful aspect... I think a point I mentioned earlier, is that a paediatric patient reminds you of the natural state of the body, uhm, and brings you back to the fact that the body was designed to heal itself. So, if the patient is not responding favourably quite early on, it is then the best to refer uhm, and we discussed that at length at one of the lectures with Dr Kateland back in the day. Uhm, other than that... No, I do think that we were quite limited resource wise. Uhm, there weren't any other textbooks that were provided or recommended, other than that sort of coursework structure in the form of a booklet that we received." (Participant 6)

The majority of participants stated that the practical component of the paediatric module was the most beneficial in preparation for paediatric assessment, management and treatment. Discontent however, was voiced regarding the limited content and scarcity of the paediatric practical component. This is supported by the following participant's opinion:

"I think well, in my year the paediatrics course was very theoretically based. There were very little practical components, but subsequent to that, when I was finishing up my research, I attended other classes and they were 
more practically orientated, were there were kids brought in. We were doing examinations on kids and you know, just looking at the body type and the way to manage a paediatric patient and I think that was the most interesting bit for me. It was not my class, so I wasn't very heavily involved, but just to observe that and to see the difference in just learning something in theory and then actually applying it. Uhm that was very interesting and uhm, certainly beneficial, but I didn't have enough of that." (Participant 5)

Another participant strongly disagreed stating that no aspect of the paediatric module was useful in their clinical management of the paediatric population. This is confirmed by the following statement by one of the participants:

"There is nothing that really comes to mind (participant laughs) in this case, er, because as I said it's- there is nothing that I really found to be that useful..." (Participant 9)

\section{Additional sources of information utilised to facilitate greater knowledge consolidation during the Chiropractic paediatric module}

Diverse responses were obtained from participants on the use of additional sources of information during the paediatric module to foster a greater understanding in the field of paediatrics. The majority of participants reported no utilisation of additional sources of information, as confirmed by the following excerpts:

"Uhm, I don't think I had any additional sources. I never read up on research, uhm, I didn't have any extra textbooks or anything like that." (Participant 7)

Another participant disagreed with the following response:

"So, I remember doing geriatrics and paediatrics and finding journal articles there and Google. Yes, just the internet and other chiropractors who helped me." (Participant 10)

\section{Recommended areas of improvement in the Chiropractic paediatric module}

The responses from participants were again diverse and emphasised various aspects of improvement in the paediatric module. An early introduction to paediatric care was popular amongst some participants. This is confirmed by the following participant who said that:

"A bit more time can be put into it and I don't think it should be just reserved for fifth year." (Participant 9)

The majority of participants recommended that the module be lectured by a paediatric Chiropractor or the introduction of a variety of Chiropractors and healthcare specialists to share their knowledge and experience with the students. This is substantiated by the following excerpt from a participant who stated:

"Something, er, needs to change... Either the teaching... So were taught these Gonstead listings and what not, I would like to learn a way of assessing a child (Participant pauses) well not I would like to learn, I think it's important that what is taught is a way of assessing a child in a Diversified manner." (Participant 1)

Participants also suggested an increase in the practical sessions in the paediatric module, focusing on paediatric adjustment and assessment. This is confirmed by the following statements from participants:

"I think that a lot more practicals with the little ones would obviously be very beneficial."

"I definitely think more hands-on experience." (Participant7)

Some participants also expressed the opinion that paediatric shelters or clinic visitations could enhance the development of confidence and competence in paediatric management, which is supported by the following statement:

"I mean there is a lot of paediatric clinics around, even if it is not to adjust, it is just to practice doing the diagnostic examinations on and motion palpation." (Participant 10)

The promotion of chiropractic care for paediatrics in clinic and the public sector to increase the exposure of chiropractic students and young professionals to paediatric management was also recommended. This is supported by the following:

"Uh, and then also I think that a lot more needs to be done with er, educating the public that paediatric patients can be treated well by chiropractors. A lot of people do not know that, and I think that is part of the reason why we do not get as many paediatric patients as we would like. Uhm, and yes, the more paediatric patients that we see, the more comfortable we would be with treating them going forward..." (Participant 9)

A minority of participants felt that greater attention should be placed on identifying serious pathology in the paediatric population. This is supported by the following excerpt:

"So I definitely think that we need to go into more detail, sort of know more conditions regarding actual 
paediatrics." (Participant 4)

\subsection{Theme Three: Challenges Encountered In Paediatric Patient Management}

Parental satisfaction and influence on treatment regime, communication difficulties with paediatric patients, uncooperative patients and time management were listed as challenges by new graduate chiropractors in their treatment and management protocols of paediatrics.

\section{Parental satisfaction and influence on treatment protocol}

Parental satisfaction and influence on treatment protocol were reported as obstacles in paediatric management, which is confirmed by the following excerpt:

"Uhm, I guess getting the mother involved as well with the treatment, uhm obviously is quite challenging you know, getting the mum to hold the baby right and all those sorts of things. Uhm, management, you also er, depend a lot on the parent and that the parent is doing their job properly and bringing the kid for their consults and all those sorts of things. So you do really have to depend on the parent, uhm, to follow the treatment protocol correctly." (Participant 7)

\section{Communication barrier with the younger paediatric population}

The inability to converse with children presented a unique challenge to new graduate chiropractors, as confirmed by the following statement:

"It is difficult, er also that the child cannot convey what pain they feel or uhm, where it is sore or why they are crying." (Participant 1)

\section{Uncooperative paediatric patients}

The unwillingness to cooperate on the behalf of paediatric patients was also reported as a challenge. This opinion is shared by a participant who stated that:

“... Sometimes they do not want you touching them and sometimes they get impatient and they do not want to sit for too long, so you have to be quick in whatever you do. So I think that is the hardest thing, especially in your younger patients. " (Participant8)

\section{Time management}

Due to the need for a more thorough physical examination, time management was reported as a challenging aspect during paediatric consults, which is supported by the following excerpt:

"I generally block off more time for a paediatric case because I don't know what I'm going to see. I generally do an extremely complete workup, er, all sort of systems testing as much as possible..." (Participant 1)

\section{Knowledge, confidence and practical experience in paediatric patient management}

A lack of knowledge, confidence and practical experience was expressed as a challenge in paediatric treatment and management. This is substantiated by the following statement: "I think the challenges in the beginning were certainly uhm, lack of practical ability to treat a musculoskeletal problem in a very young child." (Participant 1)

\subsection{Theme Four: Enhancement of Paediatric Patient Management}

Participants listed paediatric assessment, treatment protocols and post-graduate course attendance as desired areas of self-improvement in their paediatric care.

\section{Improvement in paediatric assessment}

Communication, diagnostic and time management skills were reported as possible areas of improvement in the paediatric assessment. A participant sharing a similar opinion said that:

"I think it would just be being able to handle children better. This is in the younger population obviously. Being able to cope, uhm, with the various aspects that go into a case and also to be confident enough, you know, to make a functional diagnosis." (Participant 5)

\section{Improvement in paediatric treatment}

A desire to enhance the provision of therapeutic service to paediatric patients was expressed. This is outlined by a participant that stated:

"I think one is infant adjusting techniques that are congruent to my own adjusting techniques. I don't practice NIP. Uhm, I've never been taught how to practice NIP. A lot of neonatal and, uhm, paediatric chiropractors use NIP, so I would like to learn some further techniques that can be in some sort of Diversified manner to treat paediatrics." 


\section{(Participant 1)}

\section{Continuation of professional development in paediatrics}

An interest in post-graduate course attendance to enhance paediatric management was exhibited by some participants. This is supported by a participant who held the opinion that:

"I will most definitely do a post-graduate paediatric course. I just think that having a course that is specific to paediatrics, uhm, where they teach you like the tricks and the in-depth sort of things about paediatrics... Uhm and even yes, the extra tests that you can do on paediatrics. Then I think I'd be a bit more comfortable with paediatrics." (Participant 7)

Another participant disagreed stating that:

"No I wouldn't. Not really. I've learnt what I needed to from other practitioners who I have spent time with since my graduation. Paediatrics is not a particular field that I feel like going into. Uhm, I've got many other niches that I enjoy. Many others such as; family practice, geriatrics, sport, scoliosis... Uhm a lot of niches and I don't feel particularly drawn to paediatrics." (Participant 1)

A few participants divulged mixed opinions regarding post-graduate course attendance. This is supported by a participant who said:

"Uhm, so I would be interested in attending a course, however in terms of cost and availability in S.A, in that case, uhm, I might have to take a second thought at that. Especially if it is out of the country, simply because of the cost factor, but yes, if there was one available locally, I would be willing to attend." (Participant 9)

\section{Discussion}

\subsection{Theme 1: Self-Perception of Confidence, Knowledge and Skill in Paediatric Patient Management}

It is important to assess new graduate chiropractors' perceptions of their confidence and competence with regards to paediatric patient management, to determine the effectiveness of the chiropractic paediatric programme in preparing chiropractors to manage and treat paediatrics in private practice. Participants were asked questions exploring these aspects and conveyed a deficit in their knowledge and confidence with regards to their paediatric patient management, especially children under the age of ten. These findings were attributed to the inability of this paediatric age group to adequately cooperate and communicate with the chiropractor and are similar to the findings of observational studies investigating the communication between paediatricians and children (Marginean, 2017).

Todd et al. (2017) and Monrouxe et al. (2014), similarly concluded that the perceptions of chiropractic students in their preparedness for clinical practice with the younger paediatric population was negatively affected by their lack of clinical exposure and confidence in the US and New Zealand respectively. In order to overcome these challenges, Todd et al. (2017), Burford et al. (2014) and Jatana (2012) suggested the implementation of the following guidelines in the paediatric education and training of healthcare professionals:

- Frequent clinical exposure to the younger paediatric patient base.

- Undergraduate clinical placement programmes aimed at fostering confidence and greater clinical exposure.

- Paediatric emergency management training.

A strong correlation was also noted between lack of self-perceived skill in paediatric management and an increase in the referral rate of paediatrics to experienced chiropractors or healthcare professionals, such as paediatricians. These findings are congruent to those of Barnett et al. (2011), in which the rationale for referral among primary care practitioners and specialist practitioners were explored. Referral mainly occurred based on the perceived clinical expertise of a colleague; however other reasons for referral to particular practitioners included:

- Good feedback and positive previous interaction reported by the patient.

- Availability on the local area or hospital.

- Medical record access between practitioners.

- Previous referrals from the practitioner.

Vermeir et al. (2015) highlighted the importance of inter-professional communication and suggested that communication skills between healthcare professionals should be integrated to a greater extent in the undergraduate and post-graduate training phases. 


\subsection{Theme 2: Paediatric Exposure and Training received in the Chiropractic Course}

The consensus held amongst participants was that the Chiropractic paediatric module lacked in providing practical experience in the diagnostic and orthopaedic assessment, paediatric adjustment techniques and auxiliary treatment protocols. This lack of practical experience, according to the participants, negatively affected their assessment, diagnostic abilities, treatment and management of paediatrics in private practice. Participants suggested that the incorporation of methods to improve the theoretical application and provide increased exposure to paediatrics in frequent practical sessions.

These findings are aligned with those of Todd et al. (2017) and Howells et al. (2006), which concluded that greater exposure to paediatric patients during tertiary educational programmes, increases student preparedness to assess, diagnose and treat this patient population in private practice. The legalities and challenges encountered by the Chiropractic paediatric programme however, must be considered, regarding the consent for the use of paediatrics for class demonstrations and practical sessions.

Although limited in content and paediatric exposure, the majority of participants in the study found the practical component of the course to be the most beneficial aspect in their preparation for paediatric management in private practice. A minority of participants felt that the theoretical grounding they received was the most useful aspect in their management and provision of therapeutic services to paediatric patients. This finding is in contrast to Howells et al. (2006), which concluded that didactic teaching is ineffective and knowledge of skill alone does not impact on behaviour. The study explored medical practitioners' consultation skills, including their communication abilities, assessment and diagnostic techniques as well as treatment skills and concluded that these are most effectively acquired by practice, analysis and rehearsal. Two participants were of the opinion that no aspect of the paediatric course enabled them to sufficiently treat and manage a paediatric patient. This could be attributed to the large time lapse between the acquisition of theoretical knowledge and its practical application as well as lack of exposure to paediatric patients in the Chiropractic Day Clinic, where students acquire clinical experience.

Participants were probed regarding their use of additional sources of information to enhance knowledge consolidation during the Chiropractic paediatric course. The majority of participants reported no use of additional sources of information. These findings are in contrast to those of Dogruer et al. (2011) and Apuke et al. (2018) which concluded that a vast amount of university students utilise additional sources of information with particular utilisation of the internet for the following:

- To enhance peer learning and promote self-learning.

- To improve examination preparation.

- To conduct research on various topics.

- In order to broaden their scope of learning and reading.

The participants that did report the use of additional sources of information said that these sources were largely in the form of print and social media, chiropractic seminars and observations of chiropractors or other healthcare professionals. These findings were in alignment with those of Sahin et al. (2010) which highlighted the use of the internet in the classroom setting to promote access to multiple resources such as journal articles. Gikas and Grant (2013) further concluded that the use of social media provided a platform for interaction and collaboration with colleagues, which improved students' learning and inquisition abilities. The DUT library provides students access to textbooks, online theses and journal articles. Free internet is also provided by the university. The utilisation of these resources by chiropractic students may be hindered however, due to the time constraints enforced on students by a demanding fifth year curriculum and ineffective studying techniques.

Participants were then asked to provide suggestions that could improve the quality of education provided in the chiropractic paediatric course. A variety of responses were provided, which highlighted various aspects related to the amelioration of knowledge, confidence and skill in paediatric patient management, such as the following:

- The earlier introduction of the paediatric module in the Chiropractic programme to allow for greater focus on paediatric practical sessions and theoretical grounding. The DUT curriculum is currently being restructured to allow for the incorporation of the paediatric module into the fourth year curriculum.

- Creating a platform for other healthcare specialists to share their expertise with students. This concept has been introduced into the course in recent years.

- Frequent, structured practical sessions with greater paediatric exposure and application of theory.

- Paediatric shelters and clinic visits. In SA however, parents and guardians who retain their legal right 
over their child/ward, need to be consulted prior to the assessment or treatment of any paediatric and this is not always possible.

- The promotion of paediatric care in the Chiropractic Day Clinic and the public sector to increase the exposure of students and novice chiropractors to paediatric patient care.

\subsection{Theme 3: Challenges Encountered in Paediatric Patient Management}

Parental satisfaction and influence on treatment protocol were reported as challenges to paediatric patient management. Participants reported that parental perceived standard of care was crucial to continuation with and adherence to treatment. Parents were also reported to try and influence the treatment protocol. These findings are similar to those of Damm et al. (2015) which concluded that the doctor-patient-child interaction is often dominated by the parents, who tend to interfere with the doctor-child relationship, advocating a more passive role to the child. A practitioner however, must aim to build a trusting relationship with the child in order to allow for better information transfer and the creation of an active, autonomous role for paediatric patients during consults.

The inability of the younger paediatric population, such as toddlers, to express their region and cause of pain, as well as trying to communicate via the parent to the child, was reported by the majority of participants as a barrier to effective doctor-patient communication. Damm et al. (2015) reported similar findings when assessing the communication between paediatricians and paediatrics. The study concluded that without the child's input, an understanding of the character and severity of pain is complex, making it difficult to relieve that pain effectively. Levetown et al. (2008) reported that paediatric patients should be coached to develop their role as a healthcare partner, in order to enable them to enquire about their health concerns, exchange information and actively participate in their treatment.

A lack of cooperation in the younger paediatric population was also reported as a challenge, which could be attributed to chiropractors focusing largely on manual techniques, which require greater physical contact and patient cooperation. These findings were in contrast to those of Vermeir et al. (2015) and Marginean et al. (2017), which determined that poor practitioner communication skills and inability to establish good doctor-patient relationships were the leading obstacles to efficient paediatric management. Due to the possibility of underlying systemic and genetic pathologies which require greater time spent on the physical examination and workup of paediatric patients, time management was found to be a challenge to some new graduate chiropractors. This could further be attributed to poor case history elicitation skills, lack of practical experience in handling and treating paediatrics and uncooperative paediatric patients. Participants also felt that their lack of knowledge, confidence and practical expertise negatively impacted on their management of paediatrics. These findings are similar to those of Todd et al. (2017) and Hecimovich (2009) which reported similar results when investigating the knowledge, confidence and practical expertise of chiropractic students. Hospital visits during the fourth year curriculum allow for paediatric interaction; however this is limited due to restricted access to the paediatric ward. Sports and community service events also allow for paediatric interaction during the fifth and sixth year curriculum; however these initiatives can be enhanced by focusing on more paediatric orientated events.

\subsection{Theme 4: Enhancement of Paediatric Patient Management}

Participants expressed a desire to improve their assessment skills of paediatrics under ten years of age, with particular attention on their communication, diagnostic and time management skills. These findings are in alignment to those found by Todd et al. (2017) and Boysen et al. (2010), which concluded that chiropractic students were eager to enhance their paediatric communicative and diagnostic abilities. These participants felt that interaction with other practitioners, practical courses, notes, journal articles, and toys could enhance their assessment skills.

The desire for amelioration of treatment protocol with regards to paediatric adjustment and auxiliary techniques was expressed amongst participants. These findings are similar to those of Spingelblatt (2002) which concluded after conducting a cross-sectional survey on 1200 Canadian chiropractors, that the majority of participants desired to improve their paediatric training and treatment protocols. The majority of new graduate chiropractors interviewed expressed that post-graduate course attendance would aid their paediatric patient management by enhancing their knowledge, confidence, orthopaedic assessment and treatment protocols of paediatrics. These findings are in alignment with those of Gunawan et al. (2018) which found that nurses also desired to improve their competence and confidence by attending post-graduate courses. Mixed opinions were divulged by some participants regarding post-graduate course attendance due to the high cost and availability in SA. Similar findings were found in a study conducted by Cobbing et al. (2016) on physiotherapists, determined that cost, availability, family commitments and lack of motivation served as barriers to the advancement of their studies. A minority of 
new graduate chiropractors expressed no desire to attend post-graduate courses in paediatrics due to lack of interest to specialise in the field, extensive course duration and the willingness to refer complex paediatric cases to more experienced, specialised chiropractors.

\section{Limitations}

The study was conducted on new graduate chiropractors from DUT and was focused on the eThekwini Municipality and as such, their experience and perceptions may differ from those that graduated from other universities with different paediatric curriculums. Caution, is therefore advised against the generalisation of the findings, however, the findings may be useful as guidelines for curriculum reviews, lecturing and future paediatric orientated outreach initiatives. The study also explored the perceptions of new graduates in their confidence and competence in paediatric management however it did not employ methods to assess their actual knowledge and skills. It is therefore possible that although participants felt inadequately knowledgeable and skilled in paediatric management, this may not reflect that reality of their actual competence in this regard.

\section{Recommendations}

It is recommended that the paediatric module be introduced earlier in the chiropractic course to allow for greater allocation of time to paediatric practical sessions and theoretical grounding. To encourage further interest in paediatrics and enhance the skills and knowledge taught in the paediatric course, frequent opportunities should be created to allow paediatric specialists to interact with chiropractic students. Consistent, structured practical sessions focusing on the application of theory and paediatric communication should be integrated into the curriculum, along with paediatric clinic visits or clinical placement programmes. The promotion of paediatric care in the Chiropractic Day Clinic, at sports and community service events is also recommended. These opportunities may aid in increasing student exposure to paediatric patients, hence improving their confidence and competence in the management of this age category. The use of online and print media to enhance knowledge and understanding of the paediatric field must be encouraged and recommended by the lecturer. It is suggested for further research that a comparable study be conducted on a new graduate chiropractors from the University of Johannesburg to determine any further recommendations to enhance the chiropractic paediatric course in South Africa. It is also recommended that further research be employed to assess the actual knowledge and skill level of new graduate chiropractors in their paediatric management.

\section{Conclusion}

This study investigated the perceptions of new graduate chiropractors in their management of paediatric patients in the eThekwini Municipality. The findings indicate that the management of paediatric patients is perceived as an important aspect of chiropractic care, however new graduate chiropractors exhibited a lack of confidence in their knowledge and skill level with regards to the management of the younger paediatric population. The challenges encountered by new graduate chiropractors in their paediatric management were discussed along with beneficial aspects learnt during the chiropractic paediatric course which enabled chiropractors to better manage children. In general, however, new graduate chiropractors expressed that they did not feel sufficiently trained to manage all aspects of care pertaining to the younger paediatric population and suggested multiple areas of improvement to the chiropractic paediatric module to aid in the enhancement of the quality of education provided. The findings of this qualitative research exhibited many similarities with previous studies, however very few studies pertained to the field of chiropractic education and training. This study, therefore, adds a new, valuable perspective form the viewpoint of a new graduate chiropractor on areas of improvement in the chiropractic paediatric curriculum.

\section{Competing Interests Statement}

The authors declare that there are no competing or potential conflicts of interest.

\section{References}

Apuke, O. D., \& Iyendo, T.O. (2018). University students' usage of the internet resources for research and learning: forms of access and perceptions of utility. Heliyon, 4(2018) e01052. https://doi.org/10.1016/j.heliyon.2018.e01052

Barnett, M., Keating, N.L., Cristakis, A.N., O’Malley, J., \& London, B. E. (2011). Reasons for choice of referral physician among primary care and specialist physicians. Journal of General Internal Medicine, 27(5), 12-506. https://doi.org/10.1007/s11606-011-1861-z

Bhatia, A. (2015). At what point are you no longer a new graduate? Quora. Retrieved from https://www.quora.com/At-what-point-are-you-no-longer-a-recent-graduate

Boysen, J. C., Salsbury, S. A., Derby, D., \& Lawrence, D. J. (2016). A focus group survey of Chiropractic students 
following international service learning experiences. Journal of Chiropractic Education, 30(2), 124-130. https://doi.org/10.7899/JCE-15-25

Childrens' Institute. (2011). Legal guide to age thresholds for children and young people (policy document). Johannesburg: Children's Institute.

Damm, L., Leiss, U., Habeler, U., \& Ehrich, J. (2015). Improving care through better communication: Continuing the debate. Journal of Paediatrics, 166(1), 1327-1328. https://doi.org/10.1016/j.jpeds.2015.01.027

Davies, N. (2012). Chiropractic paediatrics. Retrieved from https://neuroimpulse.com/chiropractic-paediatrics/

Dogruer, N., Ramadan, E., \& Manevis, I. (2011). The use of the internet for educational purposes. Provedia, 28(1), 606-611. https://doi.org/10.1016/j.sbspro.2011.11.115

Dudovskiy, J. (2016). Purposive sampling. Retrieved from https://www.research-methodology.net/sampling/in-primary-data-collection/purposive-sampling

DUT Faculty of Health Sciences. (2019). Chiropractic Handbook. Durban: Durban University of Technology.

Ferrance, R. J., \& Miller, J. M. (2010). Chiropractic management of non-musculoskeletal conditions in children and adolescents. Retrieved from https://www.Chiroandosteo.com/content/10/1/14

Gikas, J., \& Grant, M. M. (2013). Mobile computing devices in higher education: Student perspectives on learning with cell phones, smartphones and social, media. Internet Higher Education, 19(1), 18-26. Available: https://doi.org/10.1016/j.iheduc.2013.06.002

Hart, J. (2016). Analysis and adjustment of vertebral subluxation as a separate and distinct identity for the chiropractic profession: A commentary. Chiropractic Humanities, 23(1), 48.

Hecimovich, M. (2009). Importance of Building Confidence in patient communication and clinical skills among chiropractic students. Retrieved from https://www.ncbi.nlm.nih.gov/pmc/articles/PMC2759993/\#_ffn_sectittle

Howells, R. J., Davies, H. A., \& Silveraman, J. D. (2006). Teaching and Learning Consultation Skills for Paediatric Practice. Arch Dis Child, 91(1), 367-370. Retrieved from https://doi.org/10.1136/adc.2005.073775

Levetown, M. (2008). Communicating with children and families: From everyday interactions to skill in conveying distressing information. Paediatrics, 121(1), 60-1441. https://doi.org/10.1542/peds.2008-0565

Maharaj, A. (2017). Children and young people: Current law. Retrieved from www.medicalprotection.org $>$ southafrica

Marginean, C. O., Melil, L., Chincesan, M., Muresan S., Georgesu, A. M., Suciu, N., Pop, A., \& Azanifirei, L. (2017). Communication skills in paediatrics: The relationship between paediatrician and child. Medicine, 96(43), 1-2. Retrieved 24 April, 2019, from https://doi.org/10.1097/MD.0000000000008399

Monrouxe, L.V., Grundy, L., Mann, M., John, Z., Panagoulas, E., Bullock, A., \& Mattick K. (2017). How prepared are UK graduates for practice? A rapid review of the literature 2009-2014. BMJ Open, 7(1), 1-15. http://dx.doi.org/101136/bmjopen-2016-013656

Morrow, S. L. (2005). Quality and trustworthiness in qualitative research in counselling psychology. Journal of Counselling Psychology, 52(2), 250-260. https://doi.org/10.1037/0022-0167.52.2.250

Mortazavi, M. M., Dogan, S., Civelek, E., Theodore, N., Rekate, H. L., \& Sonntag, V. K. H. (2011). Paediatric multilevel spinal injuries: An institutional experience. Child's Nervous System, 27(7), 1095-1100. Available: https://doi.org/10.1007/s00381-010-1348-y

Sahin, Y. G., Balta, S., \& Ercan, T. (2010). The use of internet resources by University students during their course projects elicitation: A case study. Journal of Educational Technology, 9(2), 234-244.

South Africa, Department of Infrastructure. (2012). Integrated Department Plan for the eThekwini Municipality. EThekwini Municipality: Government Printer.

Spigelblatt, L. (2009). Chiropractic care for children: Controversies and issues. Paediatric Child Health, 7(2), 86-89. Available: https://doi.org/10.1093/pch/7.2.85

Todd, A. J., Carroll, M. T., Russel, D. G., \& Mitchell, E. K. L. (2017). A prospective survey of chiropractic student experiences with paediatric care and variability of case mix while on clinical placement on Rarotonga. Journal of Chiropractic Education, 31(1), 14-19. https://doi.org/10.7899/JCE-16-4

Vallone, S. (2018). Developing the core of paediatric chiropractic leadership. Journal of Clinical Chiropractic 
Paediatrics, 17(3), 1478-1479.

Vermeir, P., Vandijck, D., Degroote, S., Peleman, R., Vehoeghe, K., Mortier, E., ... Vogelaers, D. (2015). Communication in healthcare: A narrative review of the literature and practical recommendations. International Journal of Clinical Practice, 69(11), 1257-1267. https://doi.org/10.1111/ijcp.12686

\section{Copyrights}

Copyright for this article is retained by the author(s), with first publication rights granted to the journal.

This is an open-access article distributed under the terms and conditions of the Creative Commons Attribution license (http://creativecommons.org/licenses/by/4.0/). 